\title{
Multi-Vector Outphasing Provides High Power, Low Harmonics
}

\author{
Alexey Tyshko \\ NATA Consulting LLC, Electrical Department \\ Coatesville, PA 19320, USA \\ Atyshko01@comcast.net
}

\begin{abstract}
This paper describes a further development of the Chireix-Doherty principle of the Linear Amplification using NonLinear Components (LINC). The proposed multi-vector topology facilitates the design of the high power, high frequency DC/AC inverters, operating with the resonant load, with the sinusoidal output waveform and easily controlled amplitude by combining the output power of multiple identical high efficiency switchmode inverters with the individual phase shift control. The targeted cancellation of the higher harmonics in the inverter output current allows using of the output series filter with wider bandwidth decreasing the inverter output impedance and improving the output voltage phase stability. The most important outcome of the inverter output current cleanup is a significant reduction of the conduction losses per switch which is extremely important for the high temperature applications.
\end{abstract}

Keywords - amplitude control, Chireix-Doherty, harmonics cancellation, multi-vector, outphasing, power combining.

\section{INTRODUCTION}

The Chireix-Doherty (outphasing) amplifiers that are used for RF amplitude modulated (AM) output stages are known for over 70 years [1], [2]. By combining two symmetrically phase shifted $(+/-\varphi)$ equal amplitude voltages V1 and V2 produced by

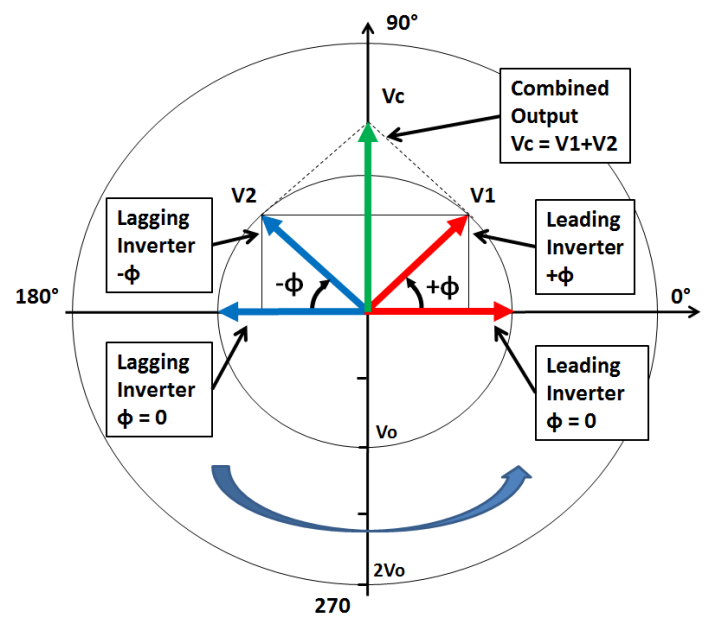

Fig. 1. Combining two symmetrically shifted $+/-\varphi$ vectors - leading V1 (red) and lagging V2 (blue) to produce the resulting vector Vc (green).

lagging and leading inverters the resulting output voltage Vc may be changed from zero to its double value compare to the original output, as shown on the phasor diagram, Fig. 1. All voltages are assumed sinusoidal. The resulting vector $\mathrm{Vc}$ amplitude depends on phase shift $\varphi$

$$
V_{c}=2 V_{Q} \sin \varphi
$$

In a process of the symmetrical modulation the resulting vector Vc stays collinear to $90^{\circ}$ axis as shown on Fig. 1 .

LINC amplifiers [3] are designed to deliver the fundamental frequency signal to the antenna using the most efficient methods of RF power generation in each of two power stages. The most efficient inverters operating in the frequency range up to a few $\mathrm{MHz}$ are switch mode ones and they produce a lot of unwanted harmonics [4].

An enhanced multi-vector topology is proposed to increase the output power and to simultaneously decrease unwanted harmonics. The principles of operation, advantages and limitations of this approach are explained based on a sample design and simulation of the AM band transmitter.

\section{TRANSMITTER REQUIREMENTS}

The transmitter function is to generate the RF signal in the resonant antenna with the capability to control output signal amplitude while keeping its phase stable - the amplitude modulation with minimum frequency modulation.

The conventional approach is based on using two phase controlled switching mode inverters $\mathrm{P} 1$ and $\mathrm{P} 2$ with the output voltage combining transformers and a load including the transmitter output Filter + Antenna [7].

The following diagrams are results of LTSpice simulations of the schematic in Fig. 3 with the arbitrarily chosen parameters: the operating frequency - $0.5 \mathrm{MHz}$, antenna voltage $1 \mathrm{kV}_{\mathrm{p}}$, antenna inductance L1 - $2 \mathrm{uH}, \mathrm{R} 1$ representing the antenna losses for Q-10 or Q-100. The antenna filter L2$1 \mathrm{uH}$ and respective $\mathrm{C} 2$ provide enough bandwidth for the spectrum of the modulation signal and possible detuning.

The simulated combined transmitter output voltage VF at the input of the filter, filter current I(L2), antenna voltage VA and L1 current I(L1) are shown on Fig. The antenna current is $90^{\circ}$ lagging to the antenna voltage. The transmitter output current, feeding the filter, is significantly smaller than the antenna current circulating in L1 and it has a lot of harmonics. 
2014 IEEE XXXIV International Scientific Conference Electronics and Nanotechnology (ELNANO)

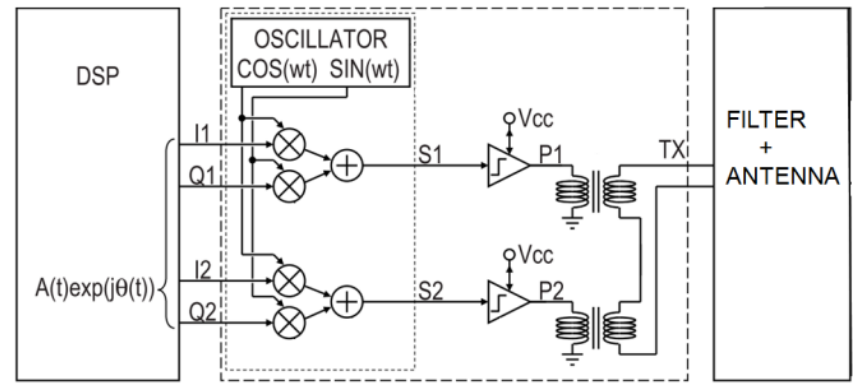

Fig. 2. Modulator and two inverter transmitter with the output combining transformers and a load including shown as Filter + Antenna [8].

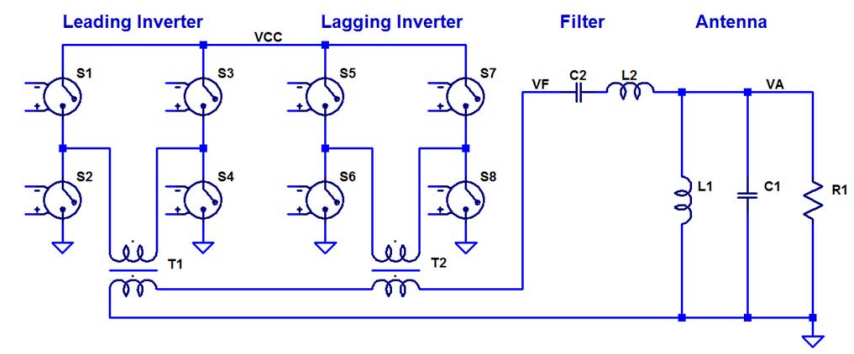

Fig. 3. Schematic used for transmitter/antenna LTSpice simulation

For simplification the control circuitry including symmetrical phase shift modulator is omitted and all attention is focused on the output voltages and output currents affecting the power switch conduction losses.

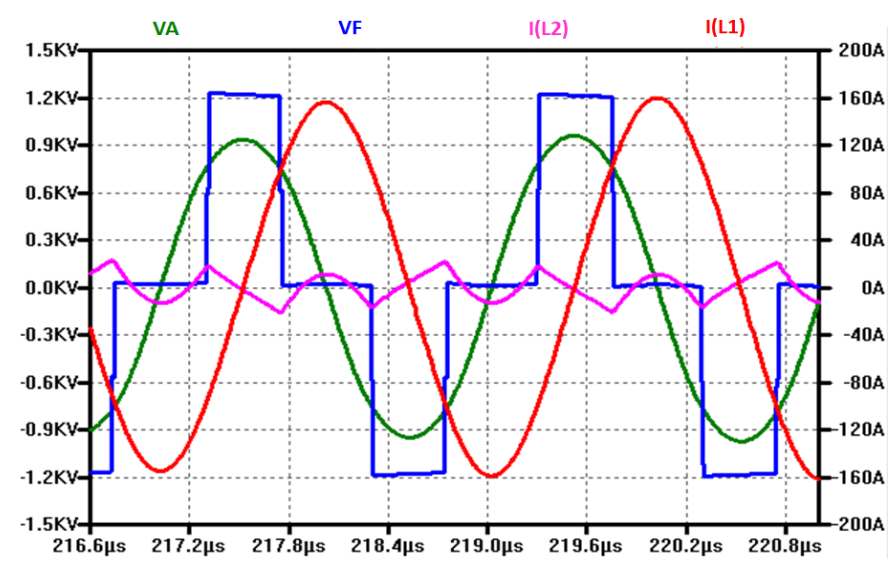

Fig. 4. Antenna voltage VA, antenna inductor current I(L1), combined transmitter output / filter input voltage VF and filter input current I(L2)

ote - the transistor switching losses may be eliminated by using ZVS mode of operation, but at expense of the increased conduction losses.

\section{SPECTRUM OF THE OUTPUT VOLTAGE}

The most efficient DC to AC switch-mode converters operate with $50 \%$ duty cycle. In this case only the odd harmonics are generated and their amplitudes $\mathbf{V}_{\mathbf{n}}$ are [4]:

$$
V_{n}=V_{o} \frac{4}{\pi n} \sin (n \times p)
$$

where $\mathbf{V}_{\mathbf{0}}$ is the amplitude of the inverter rectangular output voltage, $\mathrm{n}$ - the number of odd harmonic $(\mathrm{n}=1$ for the fundamental) and $\varphi$ - the modulation angle in degrees, Fig. 5. The relative power of the fundamental harmonic in the output voltage of the resulting rectangular voltage $\mathbf{K}_{\mathbf{1}}$ at the antenna filter input vs. modulation angle $\varphi$ is:

$$
K_{1}=\frac{F_{1}}{F_{0}}=\frac{8 \sin ^{2} \varphi}{\pi \varphi}
$$

For loads, in which the only useful power is the fundamental harmonic $50 \%$ duty cycle $\left(\varphi=90^{\circ}\right)$ is not optimal. The optimal angle is $67^{\circ}$, and often used $\varphi=60^{\circ}$ eliminating the $3^{\text {rd }}$ harmonic, as shown on Fig. 5, is very close to the optimum.

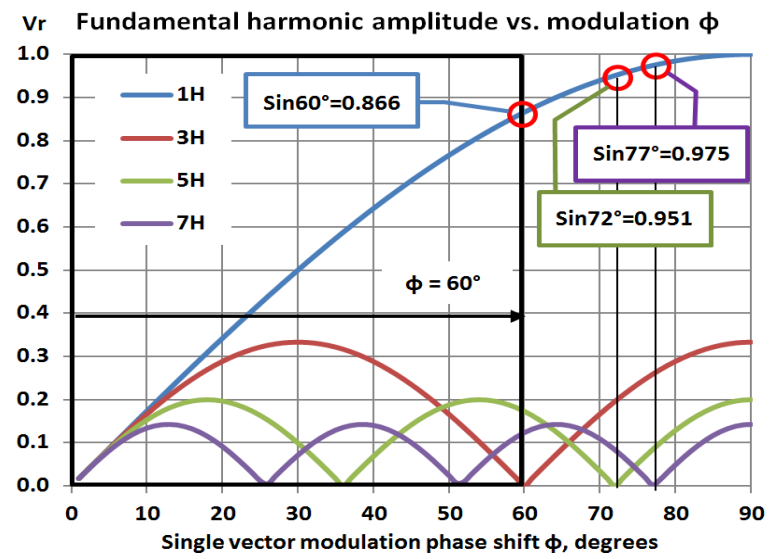

Fig. 5. Odd harmonics values vs. $\varphi$, sample values are for $60^{\circ}, 72^{\circ}$ and $77^{\circ}$.

\section{OPERATION WITH THE RESONANT ANTENNA}

The antenna LC components store the energy in both electrical and magnetic fields and dissipate this energy in resistance $\mathrm{R}$. Any change in the stored energy requires the injection of the external energy into the system or the removal of the energy from the system with the possible return of a part of this energy back to the source (the capacitive energy storage). All these transactions are passing through the transmitter, which has to be a two way DC/AC inverter. The transmitter output current $\mathbf{I}_{\mathbf{t r}}$ includes three components

$$
I_{t r}=I_{K}+I_{\Delta}+\frac{d}{d t} E_{A}
$$

where $\mathbf{I}_{\mathbf{R}}$ compensates the antenna active losses, $\mathbf{I}_{\Delta}$ compensates the mismatching current, i.e. capacitive or inductive, and the last component defines the rate of the antenna stored energy change which may be significant.

Fig. 6 shows the effect of the short pulse modulation, requiring an intensive energy exchange with the resonant load (antenna). The antenna output VA (in green) is shaped with two short (25us and 50us) Hann pulses. The resulting transmitter RF output current is shown in red. The blue line shows the averaged transmitter input current from DC bus.

If the antenna losses are low, a part of the antenna's stored energy may be returned back to the source as the transmitter If the antenna losses are low, a part of the antenna's stored 


\section{IEEE XXXIV International Scientific Conference Electronics and Nanotechnology (ELNANO)}

energy may be returned back to the source as the transmitter.

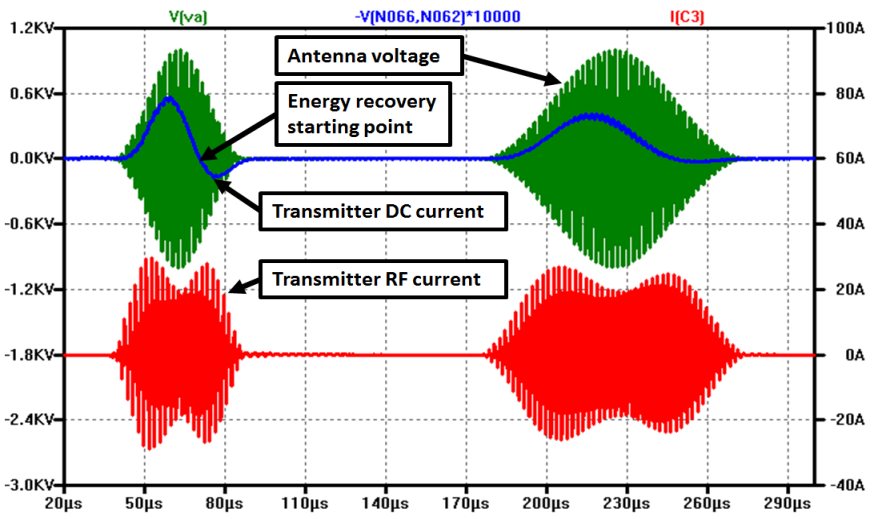

Fig. 6. Antenna voltage (green), transmitter DC current, scaled up (blue) and transmitter RF output current (red).

For the resonant load other than the fundamental current harmonics are useless. Harmonic current values $I_{n}$ are defined by their voltage $V_{n}$ and their load admittance $Y_{n}$

$$
I_{\text {out }}=\sum_{n-1} I_{n}=\sum_{n=1} V_{n} Y_{n}
$$

The resulting transmitter output current $\mathrm{I}_{\text {out }}$ is a sum of all generated harmonics. While the energy of the fundamental harmonic is transferred mostly to the load, for the higher harmonics the load (antenna) is almost a short circuit. The switching transistors and other lossy components dissipate the energy of those higher harmonics, increasing components temperature and decreasing the total system efficiency.

\section{THE RISE AND FALL OF THIRD HARMONIC}

One of the biggest problems for any switch-mode inverter is the third harmonic. For a regular electrical motor drive the presence of the third harmonic [4] significantly increases total power losses. For a transmitter driving a resonant load tuned to the fundamental frequency, the third harmonic may exceed significantly the value of the fundamental one.

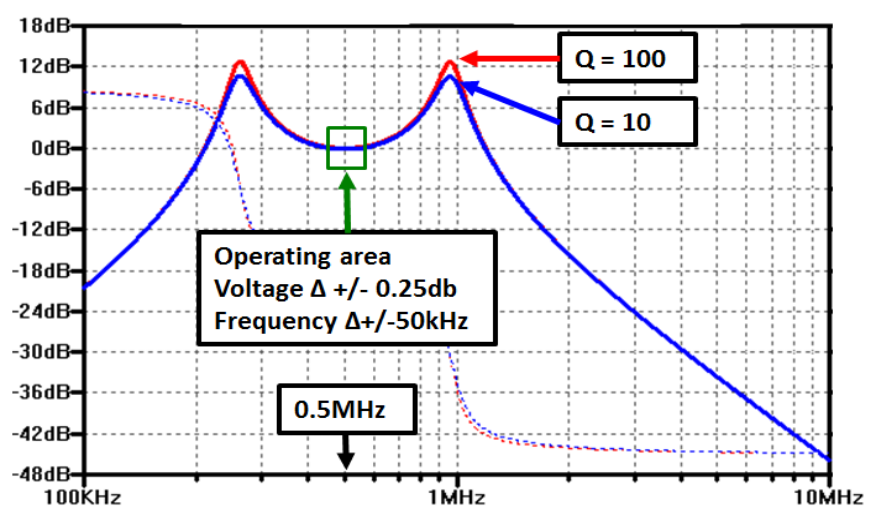

Fig. 7. Transmitter to antenna voltage transferring function.

The reason is simple: the operational area (Fig. 7, green) must be flat enough (simulated $+/-0.25 \mathrm{db}$ for $+/-10 \%$ of the operational $0.5 \mathrm{MHz}$ ) to satisfy the amplitude stability requirement for the transferring function. This may be done only by decreasing the output impedance (inductance of the tuned series filter). The resulting saddle characteristics for such wide band system are shown in Fig. 7.

Numbers $20 \mathrm{db}$ and $40 \mathrm{db}$ in Fig. 8 presenting the load admittance show the difference in the filter/antenna admittance for the $3^{\text {rd }}$ harmonic over the fundamental harmonic for the high $\mathrm{Q}$ and low $\mathrm{Q}$ antennas. If the $3^{\text {rd }}$ harmonic amplitude in the output voltage spectrum is only $10 \%$ of the fundamental, its current value may equals to the fundamental for the low $\mathrm{Q}$ or to be 10 times higher for the high Q antenna.

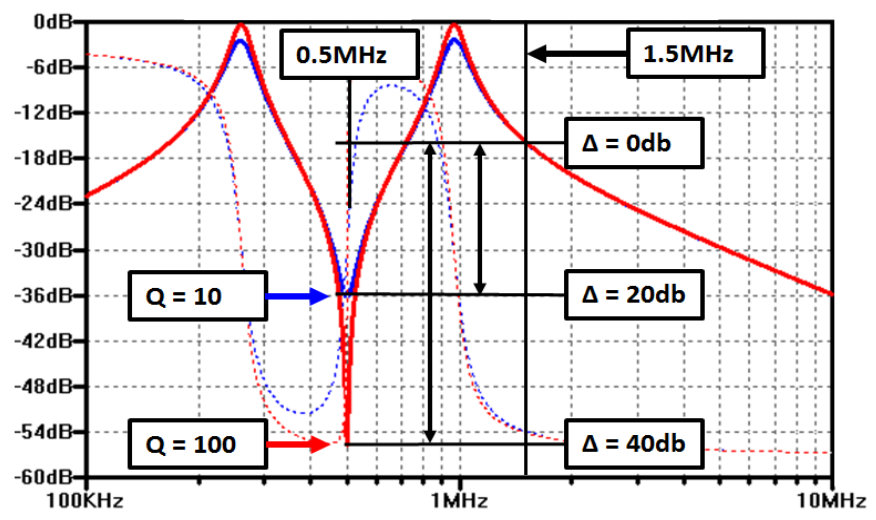

Fig. 8. Transmitter load admittance for low Q (blue) and high Q (red) antennas.

The simulation for the high Q antenna and the 25us Hann pulse envelope provides the transmitter output current spectrum and harmonic values (Fig. 9).

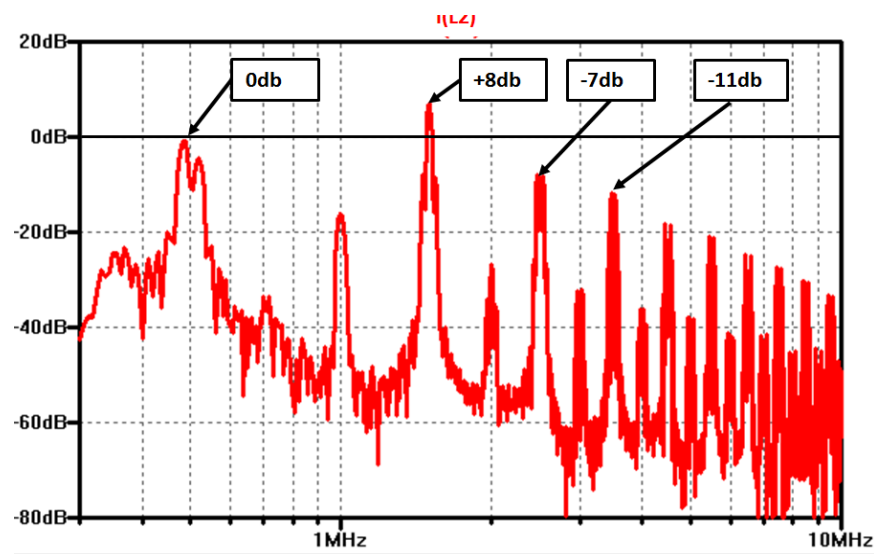

Fig. 9. Original transmitter output current spectrum for 25us pulse.

Power $\mathbf{P}_{\mathbf{s}}$ dissipated by each of the inverter switches, in the most cases MOSFET transistors, has a significant conduction loss component depending upon the drain-to-source resistance $\mathbf{R}_{\text {dson }}$ and the drain RMS current

$$
P_{s}=R_{d s e n} * \sum_{n=1} I_{n, r m s}^{Z}=R_{d s e n} * \sum_{n=1} V_{n, r m s}^{Z} Y_{n}
$$

The first odd harmonics relative power to the fundamental is shown in Table 1 for Fig. 9 spectrum with the total dissipated power 7.58 times more than for the fundamental harmonic. 


\section{IEEE XXXIV International Scientific Conference Electronics and Nanotechnology (ELNANO)}

To fix this problem the third harmonic cancellation was used in each of the two original phase controlled inverters, as shown in Fig. 10 [8]. The practical transmitter topology is presented in Fig. 11 [8]. The simulated transmitter output current spectrum is shown in Fig. 12 and the harmonic values and their power are shown in Table 1.

Table 1

\begin{tabular}{|l|l|c|c|c|c|r|}
\hline Harmonic \# & 1 & 3 & 5 & 7 & Total power \\
\hline Fig. 9 & db & 0 & 8 & -7 & -11 & \\
\hline & rel. power & 1 & 6.3 & 0.2 & 0.08 & 7.58 \\
\hline Fig. 12 & db & -2 & -24 & -9 & -13 & \\
\hline & rel. power & 0.630 & 0.004 & 0.126 & 0.050 & 0.81 \\
\hline Corrected rel. power & 1.000 & 0.006 & 0.199 & 0.079 & 1.284 \\
\hline
\end{tabular}

After $3^{\text {rd }}$ harmonic cancelling power dissipated per switch dropped almost 6 times for the same antenna power delivered.

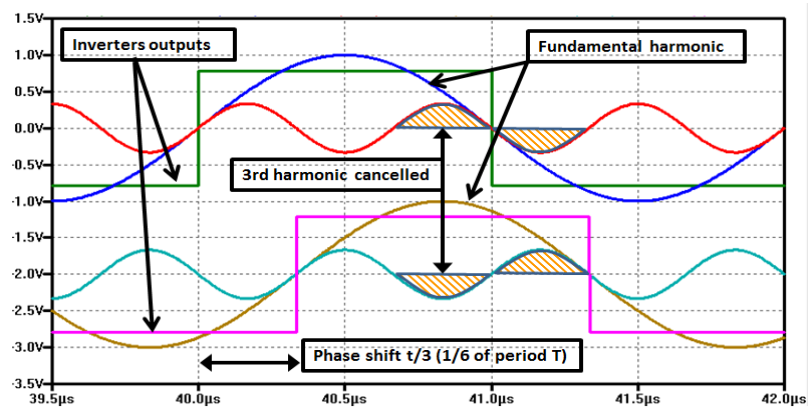

Fig. 10. Cancellation of 3rd harmonic by combining of two rectangular inverter output voltages with 60 degrees phase shift.
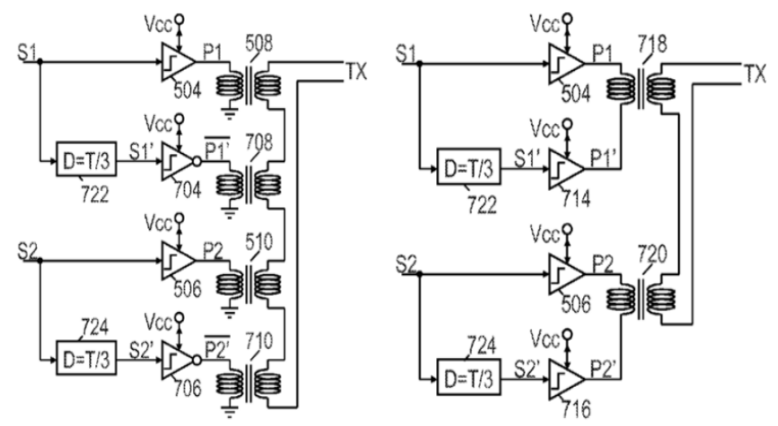

Fig. 11. Transmitter with the 3rd harmonic cancelled [8].

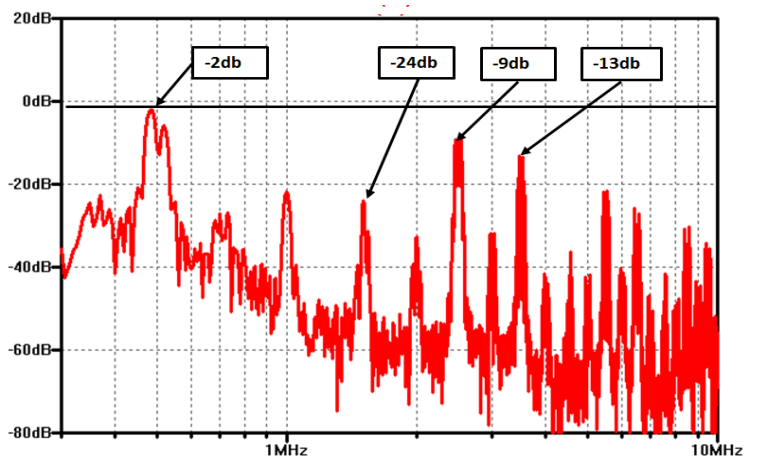

Fig. 12. Transmitter with the 3 rd harmonic cancelled output current spectrum.

Unwanted harmonics shall be cancelled prior to outphasing!

\section{MULTIPLE INVERTERS APPROACH AND HIGHER HARMONICS CANCELLATION}

The proposed approach is a multi-module, multi-vector outphasing topology, based on the following ideas:

- All modules are identical with the output transformers

- All modules are powered from the same DC power bus (input parallel connection);

- All modules AC outputs are connected in series

- Each module produces and output voltage with the same amplitude and shape (for example a rectangular voltage with $50 \%$ duty cycle)

- Each module is a four quadrant DC/AC inverter with the capability to return the load reactive current back to the power (the power source) [6]

- All modules organized in two sets forming a leading and a lagging inverters with the outputs connected in series

- Each module in the set is controlled separately by the multiple delay circuitry providing a phase shift required for the cancellation of unwanted odd harmonics at the output of each inverter (lagging and leading) as shown on Fig. 13

- The inputs of each of two delay circuits are connected to the outphasing symmetrical phase shift modulator Fig. 15

Fig. 14 shows the output voltage waveforms for different levels of the harmonic clean-up. Removed are the $3^{\text {rd }}$ (green), $3^{\text {rd }}$ and $5^{\text {th }}$ (red) and $3^{\text {rd }}, 5^{\text {th }}$ and $7^{\text {th }}$ harmonics (blue) lines.

Number of modules $\mathrm{N}$ needed to cancel $\mathrm{k}$ harmonics:

$$
N=2^{\hat{n}}
$$

where $\mathrm{N}$ is a total number of the modules, $\mathrm{k}$-the amount of odd harmonics cancelled (example: 3 removed, $\mathrm{N}=8$ ).

Value of the first harmonic $\mathbf{V}_{\mathbf{k}}$ after cancelling $\mathrm{k}$ higher ones

$$
V_{k}=V_{1} \prod_{n=3} \sin \frac{\pi}{2}\left(1-\frac{1}{n}\right)
$$

where $\mathrm{n}$ is cancelled harmonic number $-3,5,7$ etc.

The final lagging and leading inverter voltages and resulting antenna voltage are shown in Fig. 16.

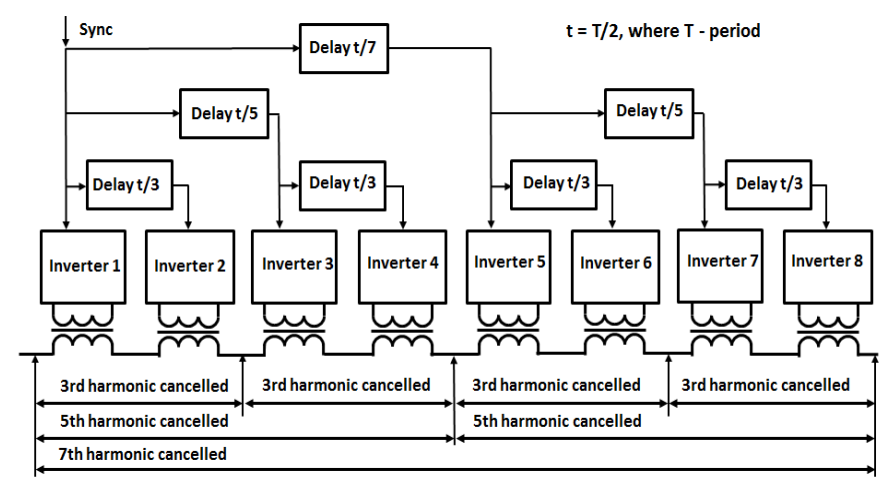

Fig. 13. Multimodule leading inverter (lagging is similar) with suppressed third, fifth and seventh harmonics in the combined output voltage, 8 modules. 


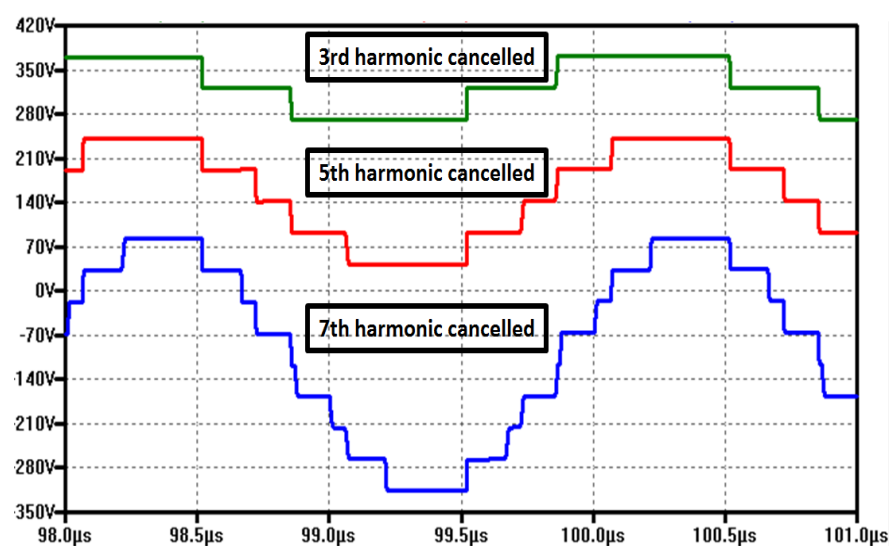

Fig. 14. Output voltage waveforms after the cancellation of the $3^{\text {rd }}, 5^{\text {th }}$ and $7^{\text {th }}$ harmonics generated with the $50 \%$ duty cycle phase shifted pulses, per set.

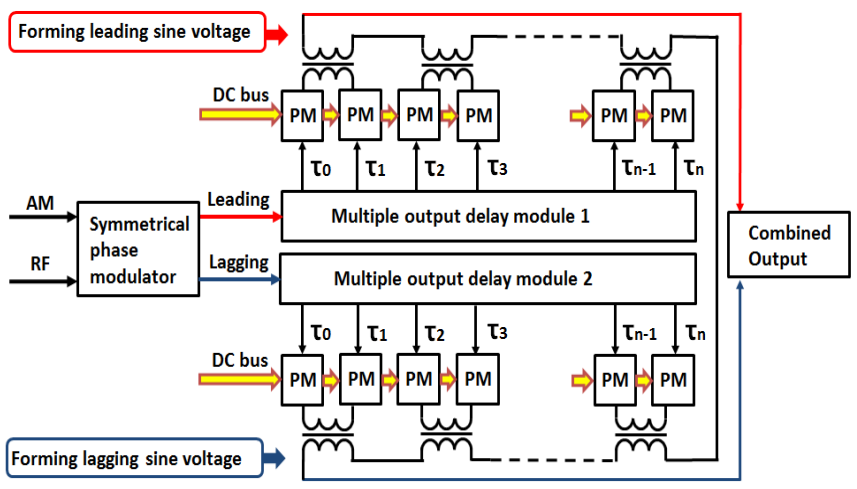

Fig. 15. Block diagram of the multistage outphasing transmitter.

Combining Leading and Lagging Inverter Outputs and Resulting Antenna Voltage

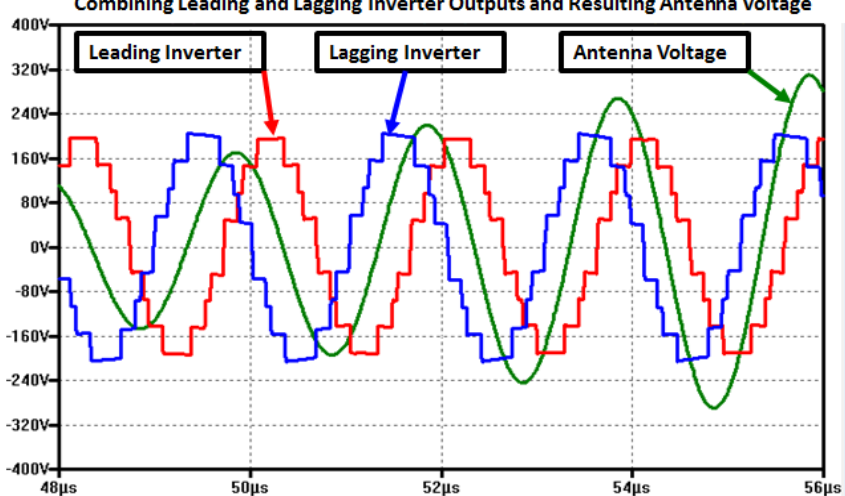

Fig. 16. Leading and lagging inverters outputs and resulting antenna voltages for a transmitter with $3^{\text {rd }}, 5^{\text {th }}$ and $7^{\text {th }}$ harmonics cancelled, total 16 modules.

With the first cancelled harmonics the transmitter output becomes cleaner as shown on Fig. 17. The nearest $11^{\text {th }}$ harmonic level is $-23 \mathrm{db}$ of the fundamental.

For harmonics far away from the series resonance at the right peak of the saddle characteristic as shown in Fig. 7 the current value $\mathbf{I}_{\mathbf{n}}$, is limited by the series filter inductance $\mathbf{L}_{\mathbf{f}}$

$$
\boldsymbol{E}_{n}=\frac{p_{y} \sin (n \varphi)}{w^{2} n^{2} f L_{f}}
$$

This means the series filter bandwidth may be increased and its impedance may be decreased while keeping unwanted harmonics and related to harmonics excessive power dissipation.

Additional advantage of the series filter reduced impedance is a better replication of the modulating signal envelope especially for short pulses with the wide spectrum.

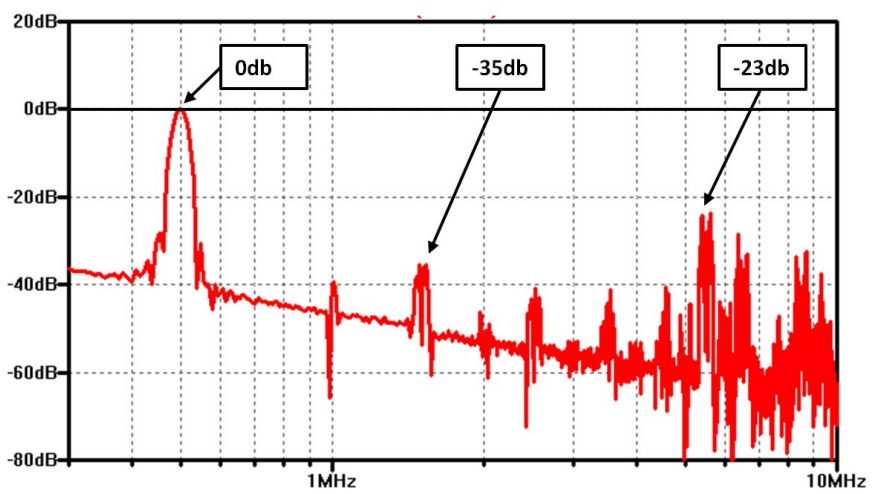

Fig. 17. Transmitter output current spectrum for a multistage outphasing transmitter with $3^{\text {rd }}, 5^{\text {th }}$ and $7^{\text {th }}$ harmonics cancelled, 25us pulse.

The choice of the optimal transmitter topology and the number of cancelled harmonics depends upon the special requirements, such as high temperature environment or increased reliability, and the practical business considerations based on the transmitter complexity resulting in the high production and service cost vs. the contract price.

\section{ACKNOWLEDGMENT}

The author acknowledges and is grateful for the methodical consultations received from Vladimir Popov, KPI, Kiev.

\section{REFERENCES}

[1] H. Chireix, "High power outphasing modulation," Proc. IRE, Vol. 23, No 11, pp. 1370 - 1392, Nov. 1935.

[2] W.H. Doherty, "A new high efficiency power amplifier for modulated waves”, Proc. IRE, Vol. 24, No. 9, pp. 1163-1182, Sept. 1936.

[3] D. Perreault, "A new power combining and outphasing modulation system for high-efficiency power amplification", IEEE Transactions on Circuits and Systems -1: Regular Papers, Vol. 58, No. 8, August 2011

[4] Power Electronics, Converters, Applications and Design. Mohan, Underland, Riobbins, 2003 Wiley \& Sons.

[5] J. Dodge, "Power MOSFET tutorial", Application Note APT-0403, Rev B, March 2, 2006

[6] G. Pavlov, A. Obrubov, "Synthesis of resonant AC converters", XIX International Conference on Power Electronics and Energy Efficiency, Alushta, Ukraine, Sept 23 - 27, 2013

[7] P. Wilkinson Dent. "Hybrid Chireix/Doherty Amplifiers and Methods", US Patent 6,133,788, Oct. 17, 2000

[8] A. Tyshko, "Enhanced Transmitter and Method for a Nuclear Magnetic Resonance Logging Tool" Patent Application US 2013/0176140 A1, Filed Jan 10, 2012, Published July 11, 2013 